\title{
Comparative dosimetric analysis of IMRT and VMAT (RapidArc) in brain, head and neck, breast and prostate malignancies
}

\author{
Mirza Athar Ali ${ }^{1}$, Muntimadugu Babaiah ${ }^{1}$, Nagaraju Madhusudhan'1, Geomcy George², \\ Shanu Jain ${ }^{3}$, Kuppuswami Ramalingam4, Sangaiah Ashok Kumar4, \\ Kalyanasundaram Karthikeyan ${ }^{4}$, Ayyalusamy Anantharaman ${ }^{4}$ \\ ${ }^{I}$ Department of Radiation Oncology, American Oncology Institute, Hyderabad, India \\ ${ }^{2}$ Department of Radiation Oncology, Advanced Cancer Diagnostic Treatment and Research Centre (ACDTRC), Bathinda, India \\ ${ }^{3}$ Department of Radiation Oncology, SGPGI, Lucknow, India \\ ${ }^{4}$ Department of Radiation Oncology, Yashoda Cancer Institute, Hyderabad, India
}

Received September 25, 2014; Revised December 16, 2014; Accepted December 18, 2014; Published Online December 23 , 2014

\section{Original Article}

\begin{abstract}
Purpose: Intensity modulated radiotherapy (IMRT) in the recent past has established itself as a gold standard for organs at risk (OAR) sparing, target coverage and dose conformity. With the advent of a rotational treatment technology such as volumetric modulated arc therapy (VMAT), an inter-comparison is warranted to address the advantages and disadvantages of each technique. Methods: Twenty patients were selected retrospectively from our patient database. Sites included were brain, head and neck, chest wall, and prostate, with five patients for each site. For all the selected patients, both the IMRT and VMAT treatment plans were generated. Plan comparison was done in terms of OAR dose, dose homogeneity index (HI), dose conformity index (CI), target coverage, low isodose volumes, monitor units (MUs), and treatment time. Results: The VMAT showed better sparing of "parotids minus planning target volume (PTV)", spinal cord and head of femur as compared to the IMRT. The lung V40 for VMAT was lower, whereas the lung $\mathrm{V}_{10}$, contralateral lung mean dose, contralateral breast mean dose and mean body dose were lower with IMRT for chest wall cases. Both the VMAT and IMRT achieved comparable HI except for the brain site, where IMRT scored over VMAT. The CI achieved by the IMRT and VMAT were similar except for chest wall cases, whereas the VMAT achieved better dose conformity. The target coverage was comparable with both the plans. The VMAT clearly scored over IMRT in terms of average MUs (486 versus 812 respectively) and average treatment time (2.54 minutes versus 5.54 minutes) per treatment session. Conclusion: The VMAT (RapidArc) has a potential to generate treatment plans for various anatomical sites which are comparable with the corresponding IMRT plans in terms of OAR sparing and plan quality parameters. The VMAT significantly reduces treatment time as compared to the IMRT, thus VMAT can increase the throughput of a busy radiotherapy department.
\end{abstract}

Keywords: VMAT; RapidArc; IMRT; Plan Comparison

\section{Introduction}

The very basic aim of radiotherapy is to deliver a tumoricidal dose to the target and at the same time spare the normal structures in the vicinity. To achieve this goal, technology is driving radiotherapy in future and that is how we have moved from three dimensional conformal radiotherapy (3D-CRT) to intensity modulated radiotherapy (IMRT) and volumetric modulated arc therapy (VMAT). IMRT in the recent past has established itself as a gold standard for organs at risk (OAR) sparing, target coverage and dose conformity. With the advent of VMAT (Rapid Arc), an inter-comparison is needed to address the advantages and disadvantages of each technique.
IMRT uses multi-leaf collimator (MLC) modulated beam delivery planned with inverse treatment planning system (TPS). IMRT treatments can be delivered by two methods. The first technique is called "Step and Shoot IMRT" in which, gantry is static at a specific angle and MLCs are static in a specific position when the beam is turned on. The second technique is called "Dynamic IMRT" in which the gantry is static at a specific angle and MLCs continuously change their positions when the beam is turned on. The classical approach to IMRT planning involves optimization of fluence maps followed by an MLC leaf sequencing step. Several investigators have proposed directly optimizing leaf positions and segment weights. ${ }^{1,2}$ In the recent past, a new technology in

Corresponding author: Mirza Athar Ali; Department of Radiation Oncology, American Oncology Institute, Hyderabad, India.

Cite this article as: Ali MA, Babaiah M, Madhusudhan N, George G, Jain S, Ramalingam K, Kumar SA, Karthikeyan K, Anantharaman A . Comparative dosimetric analysis of IMRT and VMAT (RapidArc) in brain, head and neck, breast and prostate malignancies. Int J Cancer Ther Oncol 2015; 3(1):03019. DOI: $10.14319 /$ ijcto.0301.9 
radiotherapy called Rapid Arc - a type of VMAT technique has emerged. VMAT dose optimization employs aperture based method that incorporates MLC leaf positions and MU weights as optimization parameters. VMAT is a rotational radiotherapy modality with dynamic MLC and varying dose rate. VMAT planning process optimises gantry rotation speed, dose rate, and MLC positions to create a plan. It delivers radiation with multiple superimposing coplanar or non-coplanar arcs. Hence, during the delivery of a VMAT plan, gantry continuously rotates, dose rate keeps changing and MLCs continuously move when the beam is on.

We performed a comparison of VMAT (RapidArc) treatment plans with IMRT plans in terms of OAR doses and various dose parameters which reflect the quality and delivery efficiency of a plan. Various studies have shown that VMAT may provide plan improvements for cervix and intracranial tumors. ${ }^{3,4,5,6}$ Several authors have also compared VMAT with tomotherapy. ${ }^{7,8}, 9$ Alvarez-Moret et al. ${ }^{10}$ evaluated VMAT with Oncentra Master Plan and compared with IMRT for the treatment of head and neck cancer.

A multi-institutional comparison of VMAT vs. IMRT for head-and-neck cancer showed superior VMAT plan quality parameters. ${ }^{11}$ Stieler et al. ${ }^{12}$ compared IMRT with VMAT in head and neck cancers and validated the dosimetric accuracy of VMAT. Johnston et al. ${ }^{13}$ compared simultaneous integrated boost plans generated with IMRT and VMAT for head and neck cancers and concluded that VMAT achieves comparable plans to IMRT and uses two-thirds less monitor units. Palma et al. ${ }^{14}$ compared VMAT with IMRT for prostate cancer and found more favourable dose distributions with VMAT. Quan et $a .^{15}$ reported superior plan quality as well as delivery efficiency of VMAT in prostate cancer. Zhang et al. ${ }^{16}$ showed that VMAT reduces beam on time by up to $55 \%$ as compared to five-field IMRT for prostate cancer. Rao et al. ${ }^{17}$ compared VMAT with IMRT for prostate, head-neck, and lung cancer cases. They reported $40 \%$ reduction in treatment time with VMAT while maintaining the plan quality for all the sites studied. Ali et al. ${ }^{18}$ have compared VMAT with IMRT for pancreatic cancer and Rana et al. ${ }^{19}$ have compared proton therapy with VMAT and IMRT for lung cancer. Oliver et al. ${ }^{20}$ have studied Rapid Arc and IMRT on virtual phantoms.
For the present study, sites which were considered for plan comparison include brain, head and neck, chest wall, and prostate malignancies. These cases were selected to represent a large range of clinical and anatomical complexity. The primary aim of this study is to compare the plan quality and treatment efficiency parameters of IMRT and VMAT (Rapid Arc) for different anatomical sites in order to weigh the benefits and drawbacks of each of these two high precision radiotherapy techniques.

\section{Methods and Materials}

A total of 20 patients including brain, head and neck, chest wall and prostate malignancies were selected with five patients of each anatomical site. Both VMAT and IMRT plans were generated for each patient based on identical anatomic contours, dose prescriptions and planning objectives.

Anatomical site brain included World Health Organization (WHO) grade III and IV glioma (post-surgery). Head and neck site included base of tongue and hypo-pharyngeal malignancies. Chest wall site included post-mastectomy patients requiring adjuvant radiation therapy. Pelvis site included high risk prostate cancer patients requiring radiation therapy to prostate, seminal vesicles and pelvic nodes. Table 1 demonstrates the dose prescription used for treatment planning.

All the patients were immobilized in orfit thermoplastic cast (orfit with vacloc for chest wall and prostate cases). Planning CT scan was done using Siemens dual source 64 slice computed tomography (CT) simulator (Siemens Medical Solutions, Malvern, PA, United States) and $3.0 \mathrm{~mm}$ CT slices were obtained. CT images were imported in DICOM format to Eclipse TPS, version 8.9 (Varian Medical Systems, Palo Alto, CA, United States). Target volumes and OARs were contoured. For each patient, both the IMRT and VMAT treatment planning was done using Eclipse TPS (version 8.9) and plans were computed using Anisotropic Analytic Algorithm (AAA) dose calculation algorithm. The grid size utilized for dose calculation was $0.25 \mathrm{~cm}$.

TABLE 1: Radiation dose prescription for four different anatomical sites.

\begin{tabular}{|c|c|c|c|c|c|}
\hline S. No & Site & Target & $\begin{array}{c}\text { Dose prescribed } \\
\text { (cGy) }\end{array}$ & $\begin{array}{c}\text { Dose per fraction } \\
\text { (cGy) }\end{array}$ & $\begin{array}{c}\text { Number of } \\
\text { fractions }\end{array}$ \\
\hline \multirow{2}{*}{1} & Brain & PTV 1 (Phase 1) & 4500 & 180 & 25 \\
\hline & (Sequential boost) & PTV 2 (Phase 2) & 1440 & 180 & 8 \\
\hline \multirow{3}{*}{2} & \multirow{3}{*}{$\begin{array}{l}\text { Head and Neck(simultaneous } \\
\text { integrated boost) }\end{array}$} & PTV-66 & 6600 & 200 & \multirow{3}{*}{33} \\
\hline & & PTV-59.4 & 5940 & 180 & \\
\hline & & PTV-54 & 5400 & 163.6 & \\
\hline 3 & Chest wall & PTV-50 & 5000 & 200 & 25 \\
\hline \multirow{3}{*}{4} & \multirow{3}{*}{ Prostate (Sequential boost) } & PTV 1 (Phase 1) & 5000 & 200 & 25 \\
\hline & & PTV 2 (Phase 2) & 600 & 200 & 3 \\
\hline & & PTV 3 (Phase 3) & 2200 & 200 & 11 \\
\hline
\end{tabular}

Abbreviation: PTV = planning target volume 
Multiple planners were involved in plan generation. During the process of plan optimization, planners adjusted the dose volume constraints in order to respect the OAR dose tolerance. Table 2 shows the dose volume constraints of OAR utilised for treatment planning. Table $\mathbf{3}$ shows beam geometry of IMRT and VMAT (Rapid Arc) plans for the four sites. All the VMAT (Rapid Arc) plans were performed with 2 coplanar arcs. Vieillot et al..$^{21}$ compared double arc with single arc VMAT (Rapid Arc) in anal canal cancer and found that double arc plans resulted in superior target coverage, dose homogeneity and conformity. Similar comparison study between single and double arc Rapid Arc plans was done by Kumar et al. ${ }^{22}$ for head and neck cancers.

TABLE 2: Dose volume constraints utilised for radiotherapy planning.

\begin{tabular}{|c|c|c|c|c|}
\hline S. No. & $\begin{array}{l}\text { Organ at Risk } \\
\text { (OAR) }\end{array}$ & $\begin{array}{l}\text { Dose maxi- } \\
\text { mum (cGy) }\end{array}$ & Volume & $\begin{array}{l}\text { Dose } \\
\text { (cGy) }\end{array}$ \\
\hline 1 & Bain Stem ${ }^{23}$ & $<5400$ & $1-10 \mathrm{cc}$ & $<5900$ \\
\hline 2 & Lens $^{24}$ & $<700$ & - & - \\
\hline 3 & Retina $^{24}$ & $<4500$ & - & - \\
\hline 4 & Optic nerve $e^{23}$ & $<5500$ & - & - \\
\hline 5 & Optic chiasm $^{23}$ & $<5500$ & - & - \\
\hline 6 & Parotid $^{23}$ & - & Mean & $<2500$ \\
\hline 7 & Spinal cord ${ }^{24}$ & $<4500$ & - & - \\
\hline 8 & Cochlea $^{23}$ & - & Mean & $<4500$ \\
\hline 9 & Ipsilateral lung & - & $<20 \%$ & 2000 \\
\hline 10 & Heart $^{23}$ & - & Mean & $<2600$ \\
\hline 11 & $\begin{array}{l}\text { Contralateral } \\
\text { breast }\end{array}$ & - & Mean & $<300$ \\
\hline 12 & Rectum $^{23,24}$ & - & $\begin{array}{l}<20 \% \\
<35 \%\end{array}$ & $\begin{array}{l}7000 \\
6500\end{array}$ \\
\hline 13 & Bladder $^{23}$ & - & $\begin{array}{l}<35 \% \\
<50 \%\end{array}$ & $\begin{array}{l}7000 \\
6500\end{array}$ \\
\hline 14 & Femoral head ${ }^{24}$ & - & $<5 \%$ & 5000 \\
\hline 15 & Bowel $^{24}$ & - & $<100 \mathrm{cc}$ & 4000 \\
\hline
\end{tabular}

TABLE 3: Geometry of treatment fields/arcs.

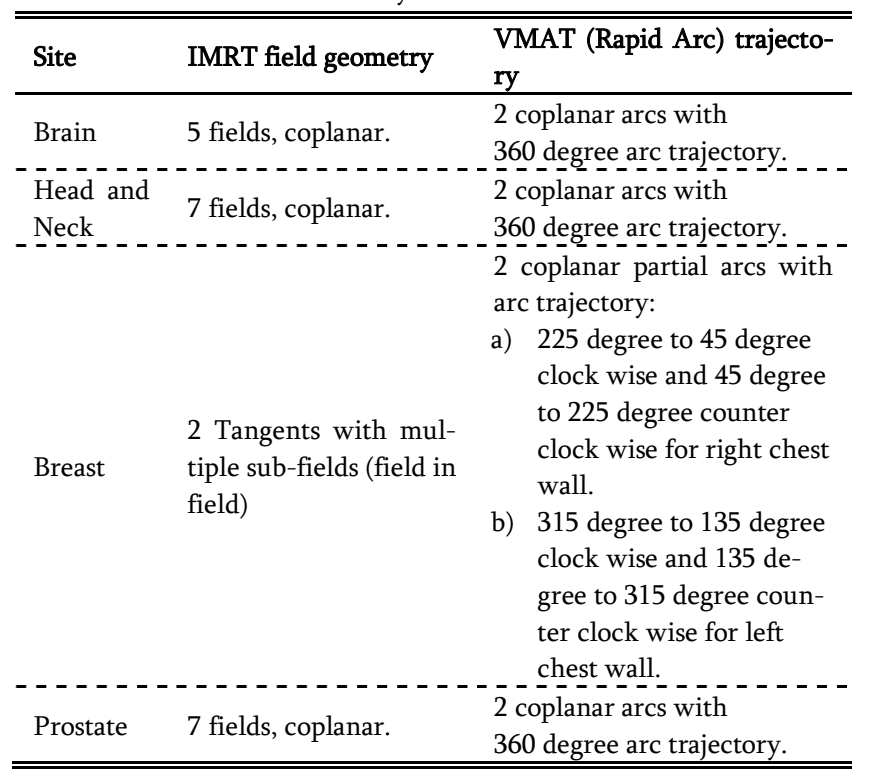

For chest wall patients, forward IMRT (field in field technique) planning was done. For cases with multiple targets, either sequential boost or simultaneous integrated boost (SIB) scheme of dose prescription was utilised. VMAT planning optimization algorithm is based on Progressive Resolution Optimizer (PRO) which uses direct aperture optimization. Because of physical limitations, upper limits were imposed on MLC leaf motion $(2.5 \mathrm{~cm} / \mathrm{sec})$, dose rate $(600 \mathrm{MU} / \mathrm{min})$ and gantry speed (5.5 degree/sec) to ensure deliverability. The linear accelerator used to deliver IMRT and VMAT plans was Varian Clinac 2100 iX (Varian Medical Systems, Palo Alto, CA, United States). Both the IMRT and VMAT plans were normalized such that at least $95 \%$ of target volume received at least $95 \%$ of prescription dose. Site specific plan comparison was performed between IMRT and VMAT plans.

\section{Plan comparison parameters}

1) OAR Dose: Mean doses were used for parallel structures and maximum doses were used for serial structures. Additional dose parameters used for plan comparison were: $\mathrm{V}_{10}, \mathrm{~V}_{20}$ and $\mathrm{V}_{40}$ (percentage volume of organ receiving respective dose in $\mathrm{Gy}$ ) for lung and heart, $V_{70}, V_{65}$ and $V_{40}$ for rectum and urinary bladder, $V_{50}$ for head of femur and $V_{40}$ for bowel.

2) Dose Homogeneity Index: $\operatorname{ImAx}_{\mathrm{M}} \mathrm{I}_{\mathrm{R}}$, where $\mathrm{ImAx}_{\mathrm{M}}$ is the maximum isodose inside the target volume and $I_{R}$ is the reference isodose (95\% isodose line). Ideally, it should be less than 2 . Lower homogeneity index value indicates a more homogeneous target dose. ${ }^{25,26}$

3) Dose Conformity Index: $V_{R} / V_{T}$, where $V_{R}$ is the reference isodose volume (volume of $95 \%$ isodose line in cubic centimetres) and $\mathrm{V}_{\mathrm{T}}$ is the volume of the target (in cubic centimetres). Ideally it should lie between 0.9 and 2.0. ${ }^{27}$

4) Target Coverage: It is defined in terms of percentage volume of target covered by prescription isodose line (95\%).

5) Low Isodose Volumes: $10 \%, 20 \%, 50 \%$ and $70 \%$ isodose volumes (in cubic centimetres) were studied to compare low dose volumes in IMRT and VMAT.

6) Monitor Units: Number of monitor units required to deliver daily prescription dose.

7) Treatment Time (beam on to beam off): Time in minutes (measured by a timer) required to deliver daily prescription dose including mode up time between fields (IMRT) and arcs (VMAT).

\section{Results}

a) For brain site (Table 4), the OAR dose sparing was better with IMRT whereas, mean whole brain dose was lower for VMAT. Figure 1(A and B) shows Dose Volume Histogram (DVH) showing comparison of OAR doses and PTV coverage, respectively. 
TABLE 4: Brain OAR doses (cGy) and plan quality parameters [Mean and standard deviation (SD)].

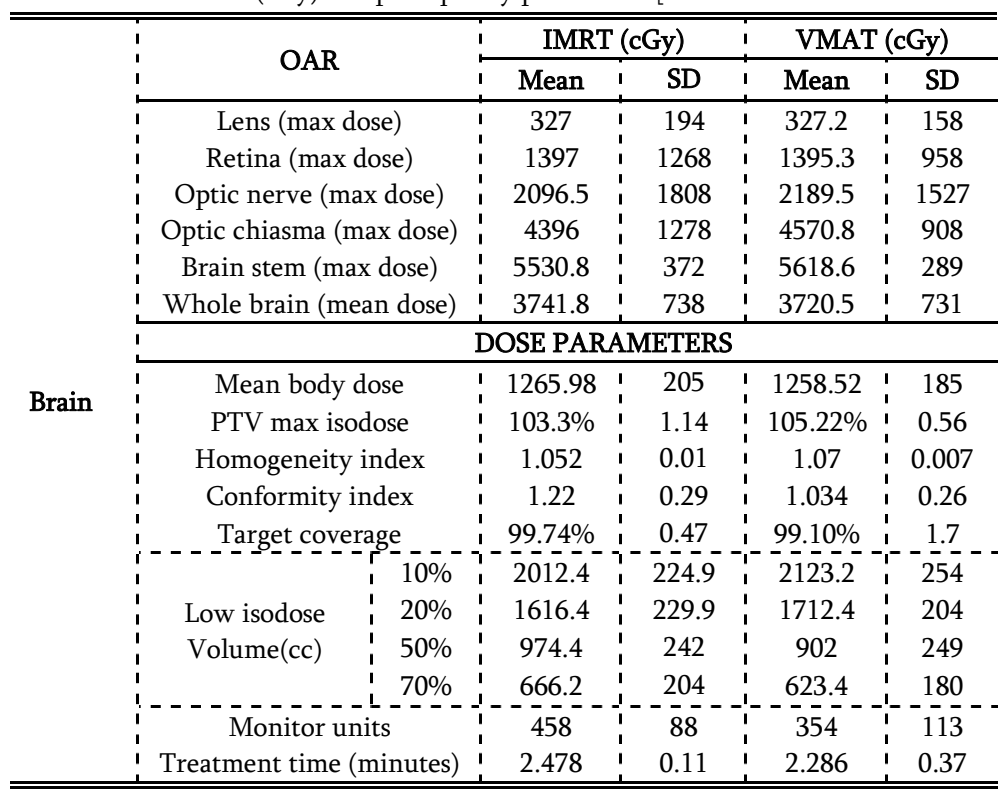

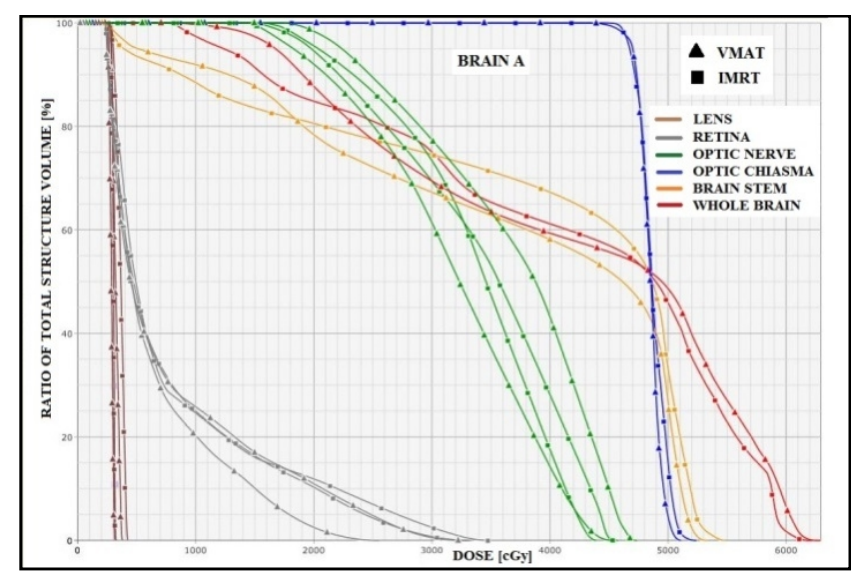

FIG. 1(A): Dose volume histogram of a brain case showing comparison of OAR doses for VMAT and IMRT.

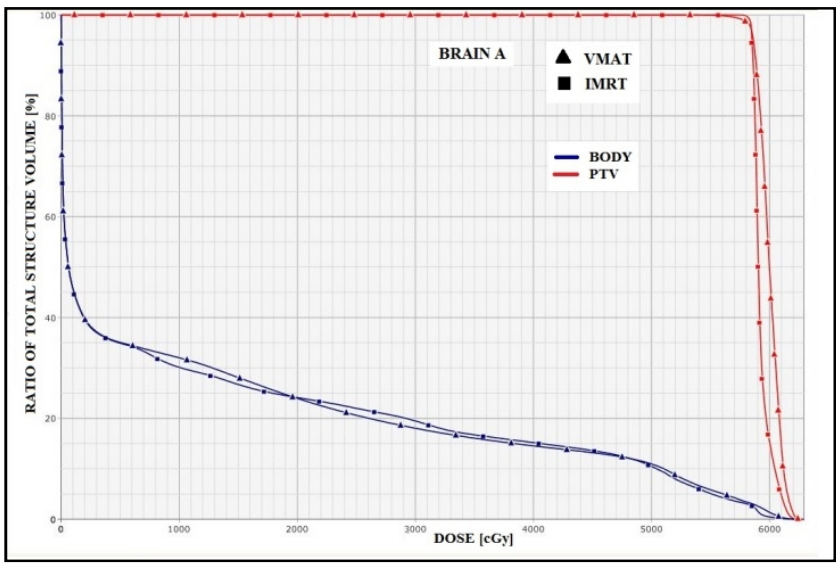

FIG. 1(B): Dose volume histogram of a brain case showing comparison of Body dose and PTV coverage for VMAT and IMRT.

b) For head and neck site (Table 5), OAR dose sparing was better with VMAT. Figure 2(B and C) shows DVH showing comparison of OAR doses and PTV coverage respectively. 
TABLE 5: Head and neck OAR doses (cGy) and plan quality parameters [Mean and standard deviation (SD)].

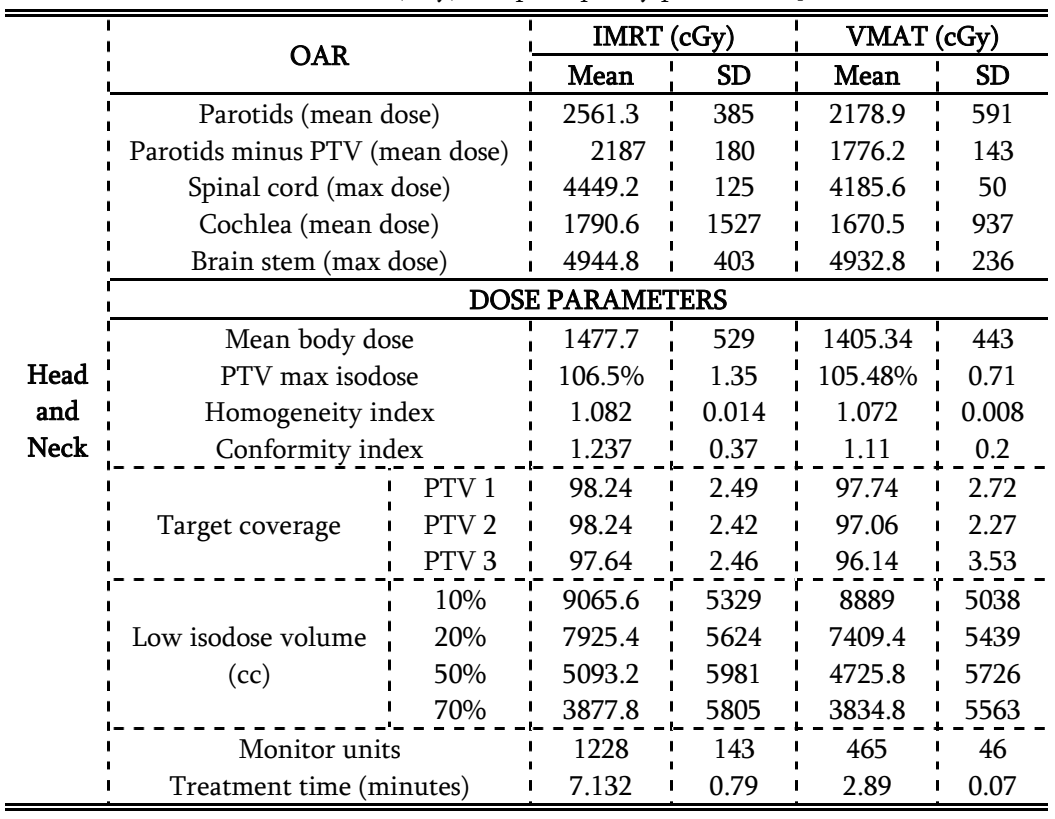
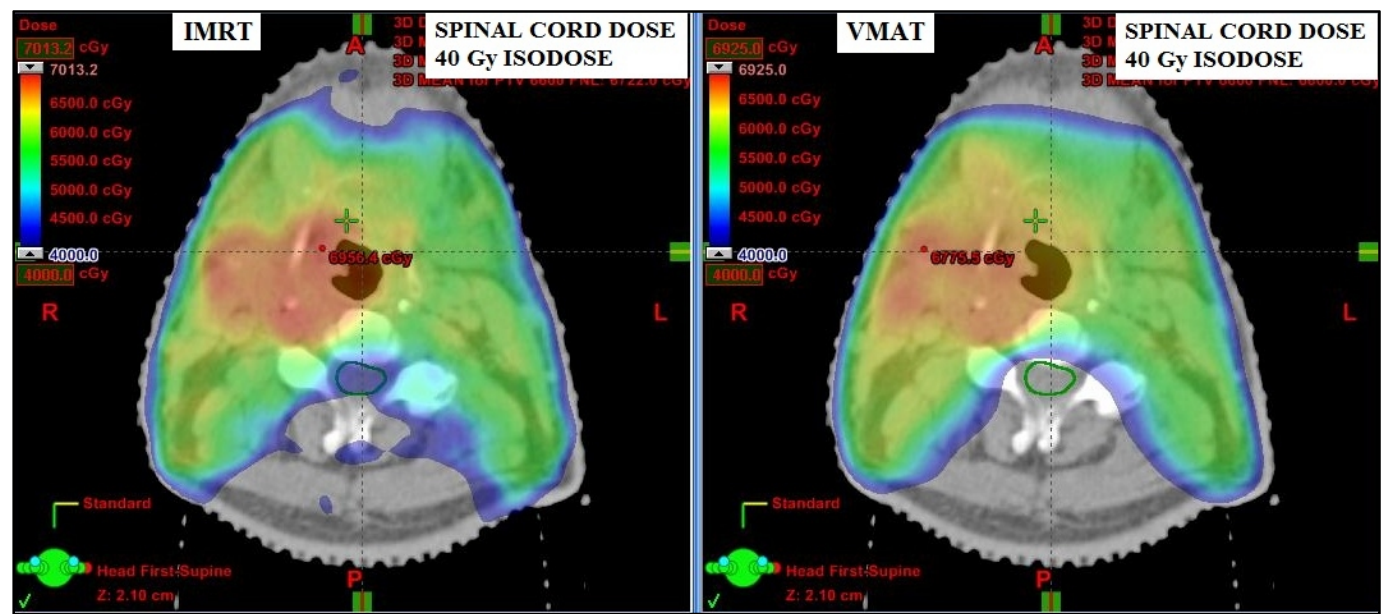

FIG. 2(A): Head and neck plan comparison: IMRT vs VMAT, axial planning CT image with dose in colour wash showing 40 Gy isodose volume.

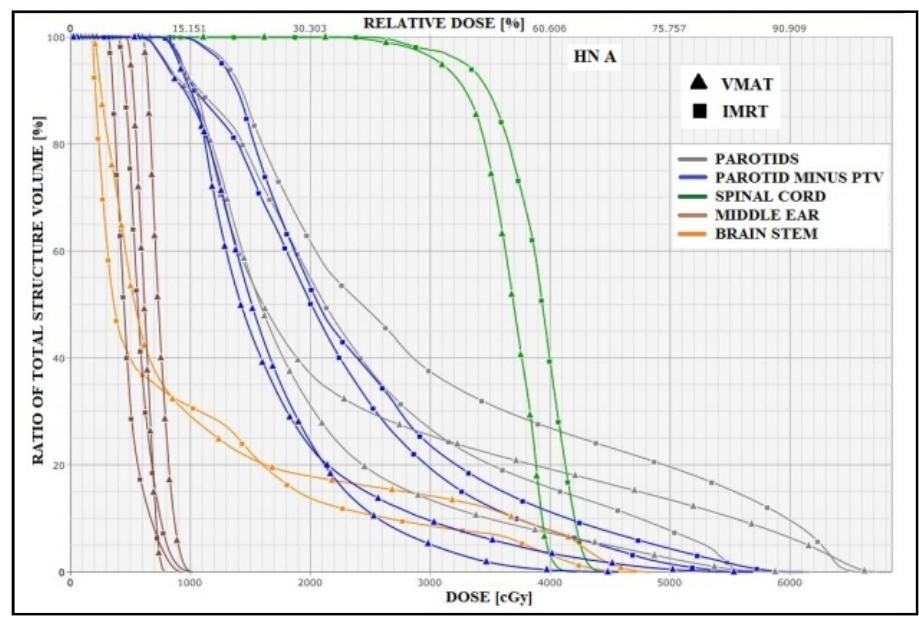

FIG. 2(B): Dose volume histogram of a head and neck case showing comparison of OAR doses for VMAT and IMRT. 


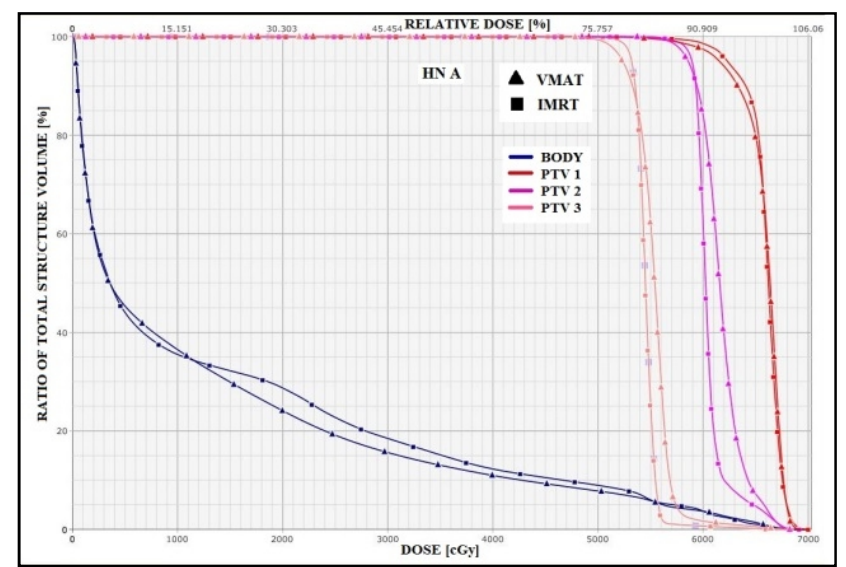

FIG. 2(C): Dose volume histogram of a head and neck case showing comparison of Body dose and PTV [PTV 1 (high risk), PTV 2 (intermediate risk) and PTV 3 (low risk)] coverage for VMAT and IMRT.

c) For chest wall site (Table 6), mean ipsilateral lung dose and $\mathrm{V}_{10}$ (Figure 3A) were lower for IMRT whereas, $\mathrm{V}_{20}$ and $\mathrm{V}_{40}$ were lower for VMAT. Mean heart dose and V10 was lower with IMRT whereas, V20 and V40 (Figure 3B) were lower for VMAT. Opposite breast sparing was better with IMRT. Figure 3C shows DVH showing comparison of OAR doses.

TABLE 6: Chest wall OAR doses (cGy) and plan quality parameters [Mean and standard deviation (SD)].

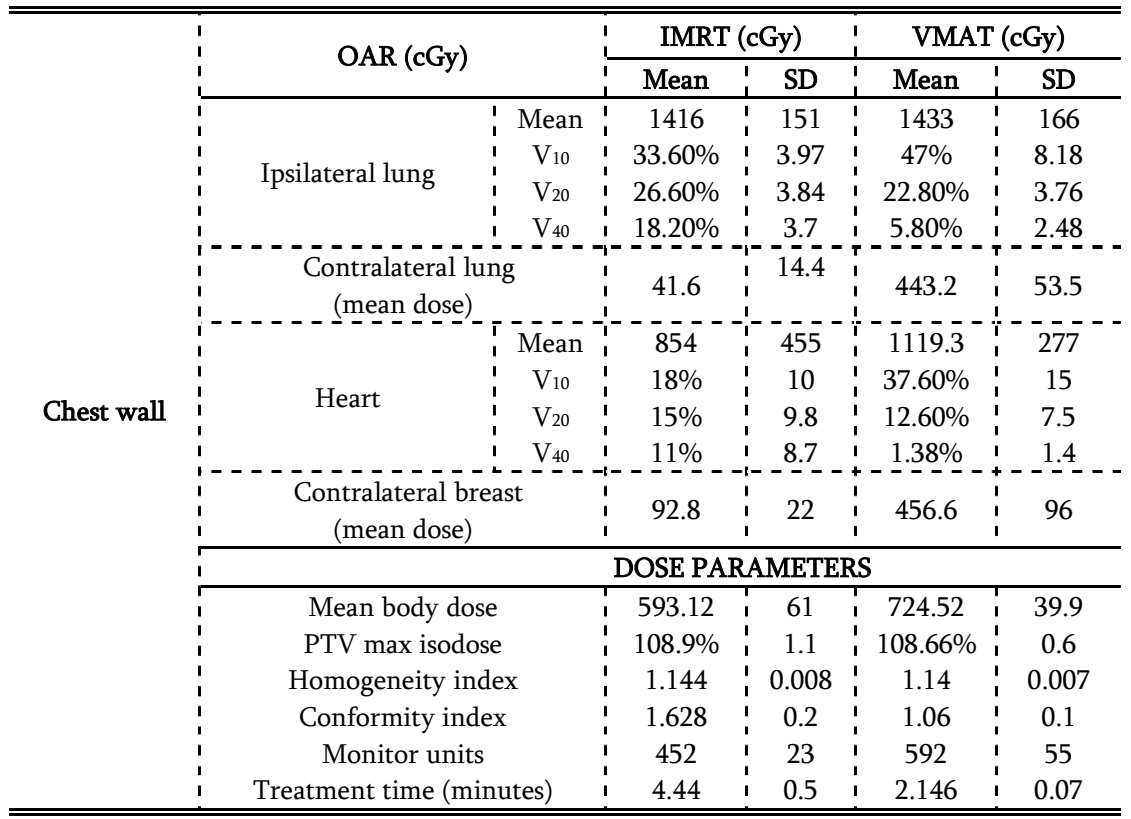
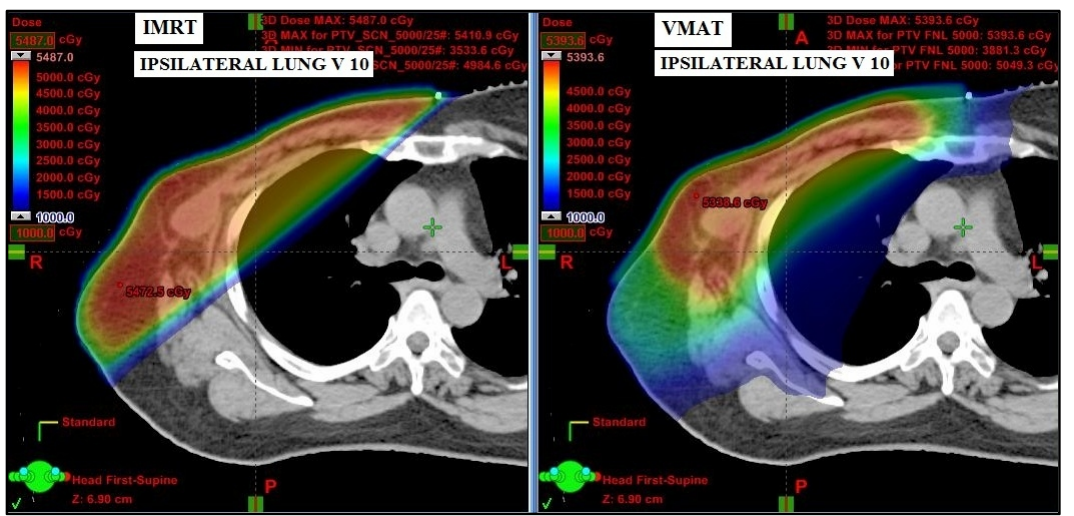

FIG. 3(A): Chest wall plan comparison: IMRT vs VMAT, axial planning CT image with dose in colour wash showing ipsilateral lung V10 (volume of lung receiving minimum dose of $10 \mathrm{~Gy}$ ). 


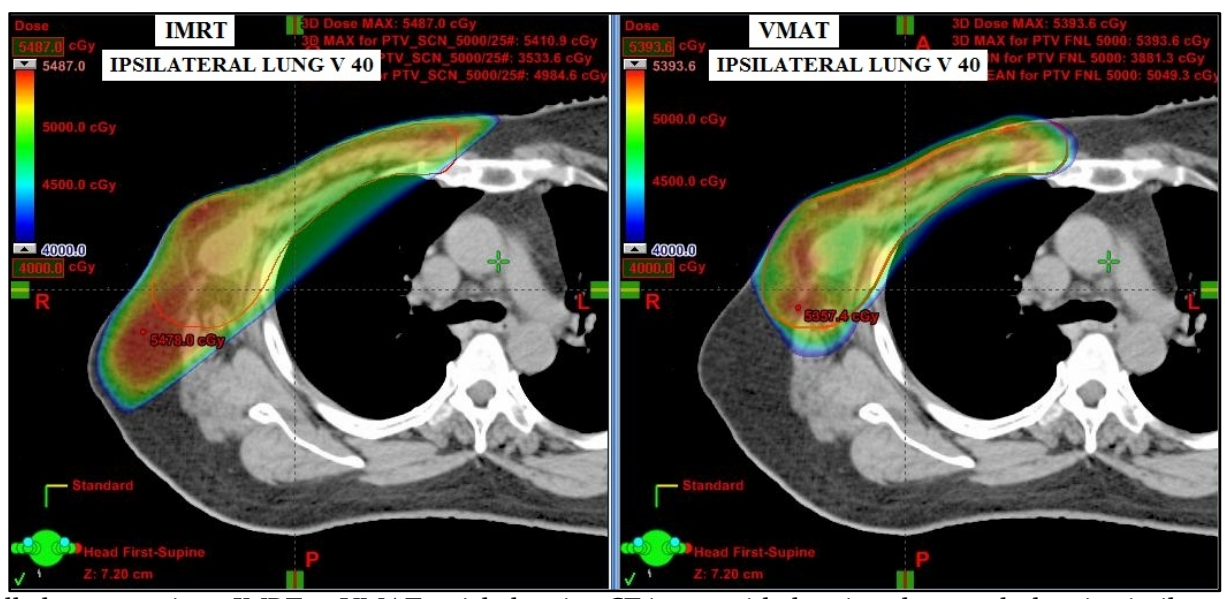

FIG. 3(B): Chest wall plan comparison: IMRT vs VMAT, axial planning CT image with dose in colour wash showing ipsilateral lung $\mathrm{V}_{40}$ (volume of lung receiving minimum dose of $40 \mathrm{~Gy})$.

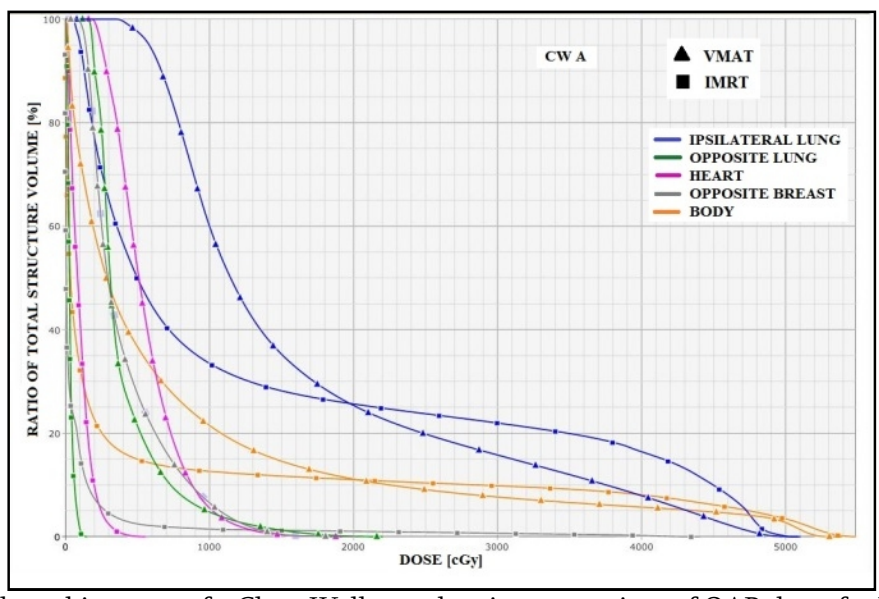

FIG. 3(C): Dose volume histogram of a Chest Wall case showing comparison of OAR doses for VMAT and IMRT.

(d) For prostate site (Table 7), rectal sparing was better with VMAT whereas, bladder sparing was better with IMRT. Femoral head (Figure 4A) and bowel sparing was better with VMAT. Figure 4(B and C) shows DVH showing comparison of OAR doses and PTV coverage respectively.

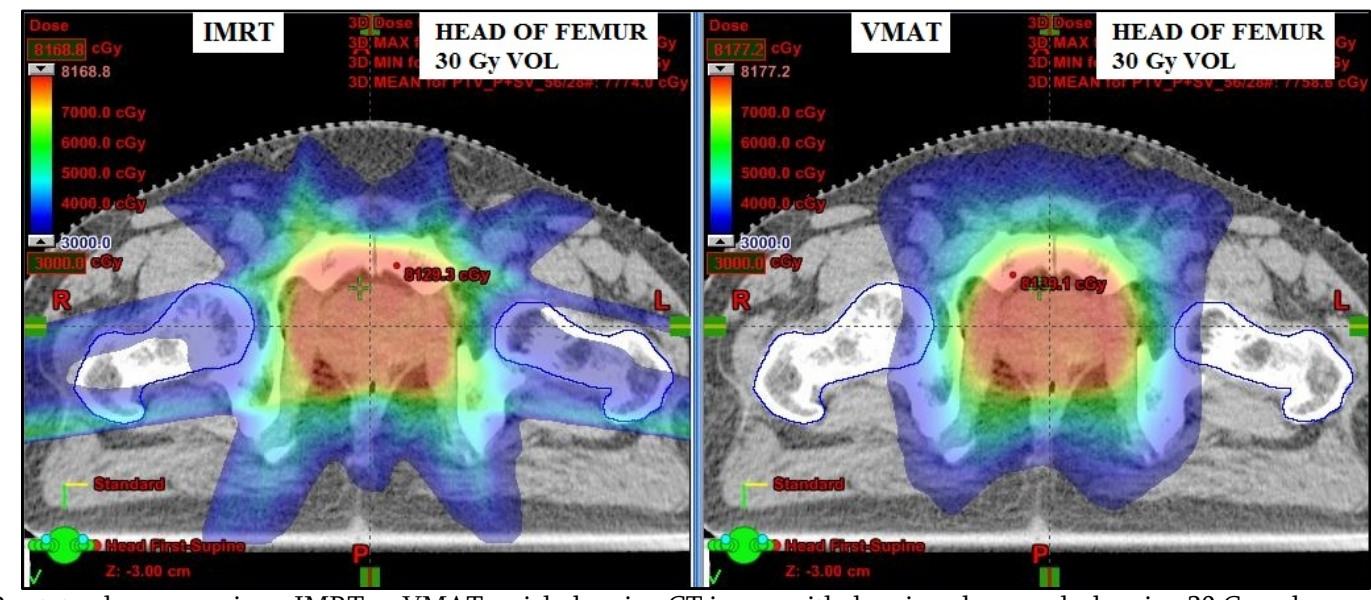

FIG. 4(A): Prostate plan comparison: IMRT vs VMAT, axial planning CT image with dose in colour wash showing 30 Gy volume and sparing of femoral heads. 


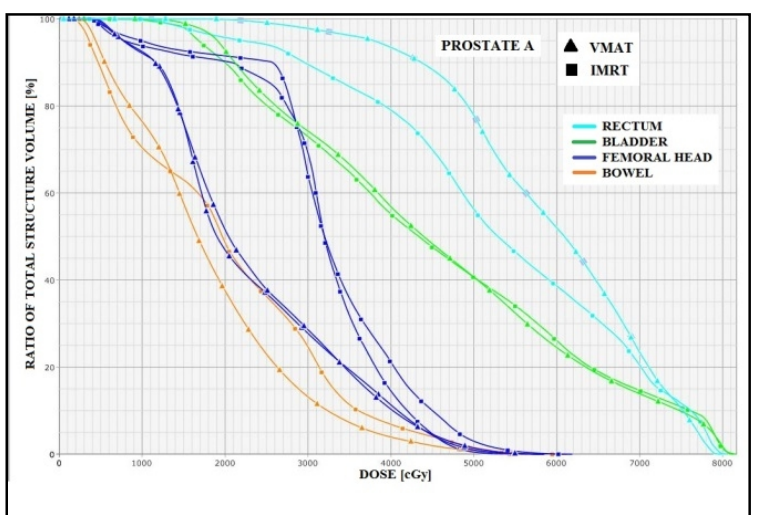

FIG. 4(B): Dose volume histogram of a Prostate case showing comparison of OAR doses for VMAT and IMRT.

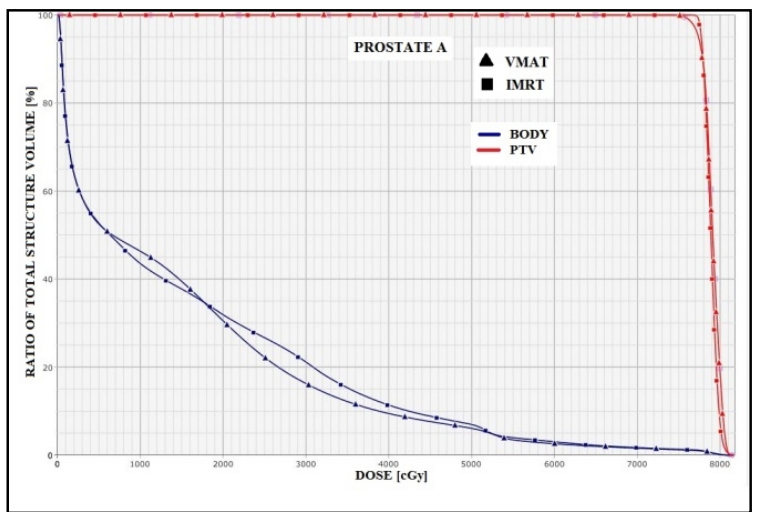

FIG. 4(C): Dose volume histogram of a Prostate case showing comparison of Body dose and PTV coverage for VMAT and IMRT.

TABLE 7: Prostate OAR doses (cGy) and plan quality parameters [Mean and standard deviation (SD)].

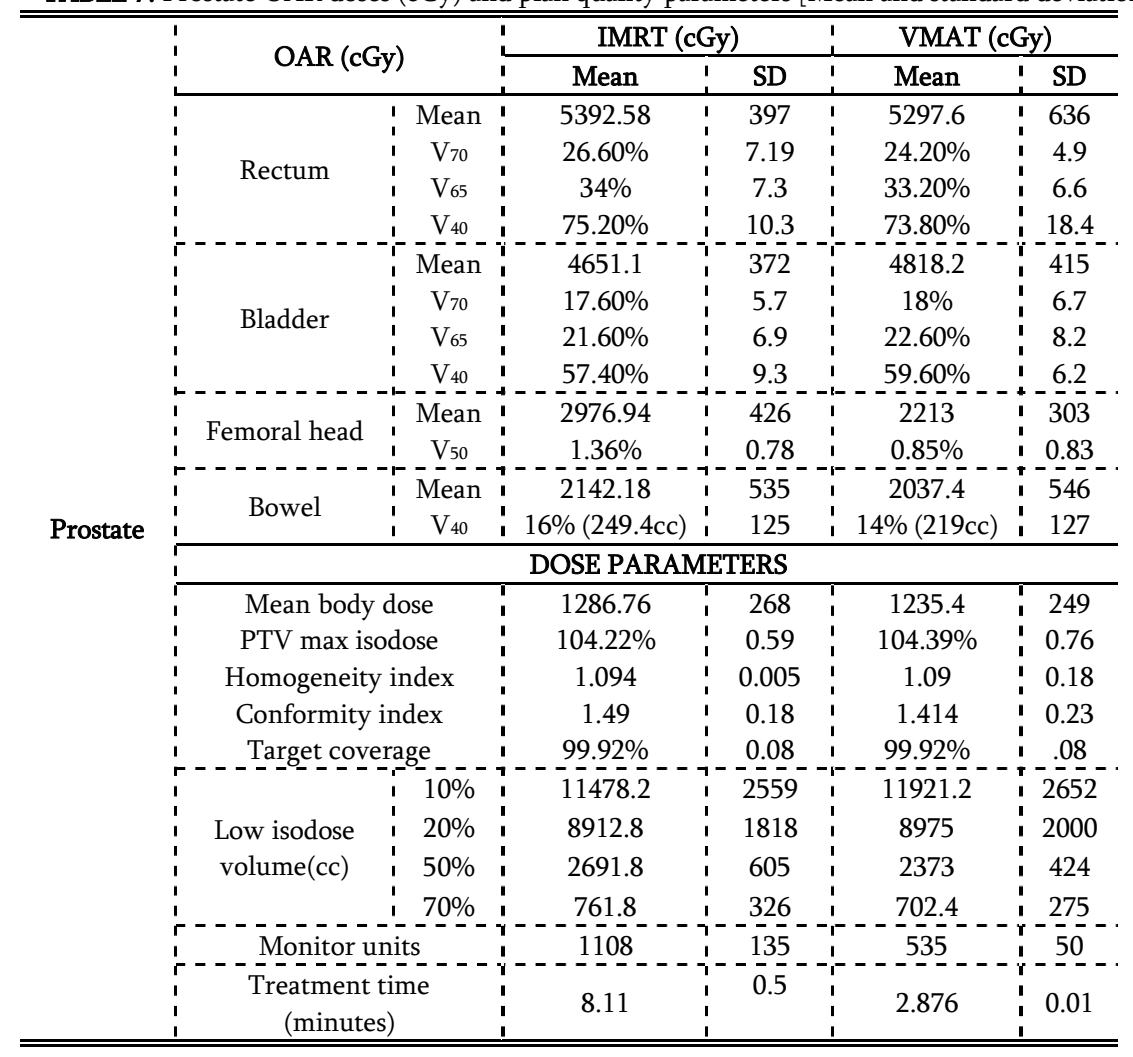


In summary, the plan quality and treatment efficiency parameter comparison shows:

a) Mean body doses for brain, head and neck and prostate were lower with VMAT whereas, for chest wall cases, mean body dose was lower for IMRT.

b) HI for head and neck, chest wall and prostate was better with VMAT whereas, it was better with IMRT for brain.

c) CI was better for all the four sites with VMAT.

d) Target coverage was better with IMRT for brain, head and neck whereas, it was similar to VMAT for prostate.

e) Low isodose volumes $-V_{10}$ and $V_{20}$ for brain and prostate were lower for IMRT whereas, $V_{50}$ and $V_{70}$ were lower for VMAT. For head and neck, all the four low isodose volumes $\left(\mathrm{V}_{10}, \mathrm{~V}_{20}, \mathrm{~V}_{50}\right.$ and $\left.\mathrm{V}_{70}\right)$ were lower for VMAT.

f) MUs required to deliver daily prescription dose for brain, head and neck and prostate were lower for VMAT whereas, for chest wall cases MUs required were lower for IMRT.

g) Treatment time with VMAT was less for all the four sites studied as compared to IMRT.

\section{Discussion}

VMAT (RapidArc) is an advanced radiation treatment modality. It has a potential to generate treatment plans for various anatomical sites which are comparable with the corresponding IMRT plans in terms of OAR sparing, plan quality with better treatment efficiency. In the present study, VMAT plans were compared with IMRT plans. Various dose-volume parameters to assess OAR sparing were studied. Plan quality was assessed by comparing dose HI, CI, target coverage, low isodose volumes, monitor units and treatment time.

It was observed that for OAR sparing, VMAT is comparable to IMRT for most of the OARs; however VMAT scored over IMRT for "parotid minus PTV", spinal cord and femoral head sparing. For chest wall cases, IMRT scored over VMAT for ipsilateral lung $\mathrm{V}_{10}$, contralateral lung mean dose, contralateral breast mean dose and mean body dose, however VMAT scored over IMRT for ipsilateral lung $\mathrm{V}_{40}$. The possible explanation for high $10 \mathrm{~Gy}$ isodose volume for VMAT is that the chest wall target is treated with a semi arc and the beam is on throughout the sweep of gantry hence increasing the exit dose through lung parenchyma. Whereas, since the IMRT plans for chest wall were all forward IMRT plans with modulation of intensity in the tangential fields (using sub-fields), only a small thickness of lung parenchyma was in the field, hence better $\mathrm{V}_{10}$.

The reason for better $\mathrm{V}_{40}$ for VMAT is that since the dose distribution was more conformal for VMAT, 40 Gy isodose line was closer to the target contour, hence minimizing the volume of lung encompassed. VMAT (Rapid Arc) showed higher $10 \%$ and $20 \%$ isodose volumes whereas, IMRT showed higher $50 \%$ and $70 \%$ isodose volumes for Brain and Prostate site. For Head and Neck site, all the isodose levels studied (10\%, 20\%, 50\% and 70\%) were higher for IMRT. Ekambaram et al..$^{28}$ analysed low dose level volumes in IMRT and 3D conformal radiotherapy.

For plan quality parameters, it was observed that VMAT and IMRT achieved almost similar dose maximum levels inside the PTV except for brain site, where IMRT scored over VMAT by achieving a lesser PTV dose maximum. Similarly, both the plans achieved comparable H.I except for brain site, where IMRT scored over VMAT because of lesser PTV dose max. C.I achieved by IMRT and VMAT were similar except for chest wall cases, where VMAT achieved better dose conformity. Target coverage was almost equally good with both the plans.

The most significant observation of this study was the difference in MUs and treatment time between the two modalities. VMAT clearly scored over IMRT in terms of MUs required to deliver daily the prescription dose. VMAT delivers significantly less MUs per treatment session as compared to IMRT. Treatment time (including mode up time) was significantly less with VMAT as compared to IMRT thereby increasing the throughput of the department. These results are in good agreement with the published literature.

The AAA calculation algorithm improves the accuracy of dose calculations over pencil beam algorithm and particular progress has been made with respect to the penumbra and low dose regions. ${ }^{29}$ However, AAA dose calculation algorithm has some limitations when there is presence of tissue heterogeneity. In general, AAA over predicts the dose beyond low density regions and under predicts the dose distal to high density tissue. In the presence of tissue heterogeneity, there can be differences of up to $10.2 \%$ in lung with AAA calculation as compared to Monte Carlo calculations. ${ }^{30}$ More recently, Acuros XB dose calculation algorithm has been introduced the results of which were found to agree better with Monte Carlo calculations as compared to AAA. ${ }^{30,31,32}$

\section{Conclusion}

The dosimetric plan quality parameters of VMAT are comparable with IMRT plans. OAR sparing with VMAT is comparable with IMRT except for "parotid minus PTV", spinal cord and head of femur, where VMAT proved itself better than IMRT. The most significant differences between VMAT and IMRT plans were the daily treatment time and monitor units per treatment session, where VMAT scored over IMRT. Reducing the daily treatment time decreases the chances of geographic miss due to organ motion and increases patient comfort and compliance. 


\section{Conflict of interest}

The authors declare that they have no conflicts of interest. The authors alone are responsible for the content and writing of the paper.

\section{References}

1. Gersem WD, Claus F, Wagter CD, et al. Leaf position optimization for step-and-shoot IMRT. Int J Radiat Oncol Biol Phys 2001; 51:1371-88.

2. Shepard DM, Earl MA, Li XA, et al. Direct aperture optimization: A turnkey solution for step-and-shoot IMRT. Med Phys 2002; 29:1007-18.

3. Cozzi L, Dinshaw KA, Shrivastava SK, et al. A treatment planning study comparing volumetric arc modulation with Rapid Arc and fixed field IMRT for cervix uteri radiotherapy. Radiother Oncol 2008; 89:180-91.

4. Fogliata A, Clivio A, Nicolini G, et al. Intensity modulation with photons for benign intracranial tumors: A planning comparison of volumetric single arc, helical arc and fixed gantry techniques. Radiother Oncol 2008; 89:254-62.

5. Shaffer R, Nichol AM, Vollans E, et al. A comparison of volumetric modulated arc therapy and conventional intensity modulated radiotherapy for frontal and temporal high-grade gliomas. Int J Radiat Oncol Biol Phys 2010; 76:1177-84.

6. Wagner D, Christiansen H, Wolff H, Vorwerk H. Radiotherapy of malignant gliomas: Comparison of volumetric single arc technique (RapidArc), dynamic intensity modulated technique and 3-D conformal technique. Radiat Oncol 2009; 93:593-6.

7. Rong Y, Tang G, Welsh JS, et al. Helical Tomotherapy versus Single Arc Intensity Modulated Arc Therapy: A collaborative dosimetric comparison between two institutions. Int J Radiat Oncol Biol Phys 2011; 81:284-96.

8. Wolff D, Stieler F, Welzel G, et al. VMAT versus serial tomotherapy, step-and-shoot IMRT and 3-D-conformal RT for treatment of prostate cancer. Radiother Oncol 2009; 93:226-33.

9. Wiezorek T, Brachwitz T, Georg D, et al. Rotational IMRT techniques compared to fixed gantry IMRT and Tomotherapy: multi-institutional planning study for head and neck cases. Radiat Oncol 2011; 6:20.

10. Alvarez MJ, Pohl F, Koelbl O, Dobler B. Evaluation of volumetric modulated arc therapy (VMAT) with Oncentra MasterPlan for the treatment of head and neck cancer. Radiat Oncol 2010; 5:110.
11. Holt A, Gestel DV, Arends MP, et al. Multi-institutional comparison of volumetric modulated arc therapy vs. intensity-modulated radiation therapy for head-and-neck cancer: a planning study. Radiat Oncol 2013; 8:26.

12. Stieler F, Wolff D, Schmid H, et al. Comparison of several modulated radiotherapy techniques for head and neck cancer and dosimetric radiation of VMAT. Radiother Oncol 2011; 101:388-93.

13. Johnston M, Clifford C, Bromley R, Back M, Oliver L, Eade T. Volumetric Modulated arc therapy in head and neck radiotherapy: A planning comparison using simultaneous integrated boost for his pharynx and oropharynx carcinoma. Clinical Oncol 2011; 23:503-11.

14. Palma D, Vollans E, James K, et al. Volumetric Modulated Arc Therapy for delivery of prostate radiotherapy: Comparison with Intensity Modulated Radiotherapy and Three-Dimentional Conformal Radiotherapy. Int J Radiat Oncol Biol Phys 2008; 72:996-1001.

15. Quan EM, Li X, Li Y, et al. A comprehensive comparison of IMRT and VMAT plan quality for prostate cancer treatment. Int J Radiat Oncol Biol Phys 2012; 83:1169-76.

16. Zhang P, Happersett L, Hunt M, et al. Volumetric modulated arc therapy: Planning and evaluation for prostate cancer cases. Int J Radiat Oncol Biol Phys 2010; 76:1456-62.

17. Rao M, Yang W, Chen F, et al. Comparison of Elekta VMAT with helical tomotherapy and fixed field IMRT: Plan quality, delivery efficiency and accuracy. Med Phys 2010; 37:1350.

18. Ali AN, Dhabaan AH, Jarria CS, et al. Dosimetric comparison of volumetric modulated arc therapy and intensity modulated radiation therapy for pancreatic malignancies. Med Dosim 2012; 37:271-5.

19. Rana S, Pokharel S, Zheng Y, et al. Treatment planning study comparing proton therapy, Rapid Arc and intensity modulated radiation therapy for a synchronous bilateral lung cancer case. Int J Cancer Ther Oncol 2014; 2:020213.

20. Oliver M, Ansbacher W, Wayne A Beckham. Comparing planning time, delivery time and plan quality for IMRT, RapidArc and Tomotherapy. J Appl Clin Med Phys 2009; 10:4.

21. Vieillot S, Azria D, Lemanski C, et al. Plan comparison of volumetric-modulated arc therapy (RapidArc) and conventional intensity-modulated radiation therapy (IMRT) in anal canal cancer. Radiat Oncol 2010; 5:92.

22. Kumar SAS, Vivekanandan N, Sriram P. A study on conventional IMRT and RapidArc treatment planning techniques for head and neck cancers. 
Reports of Practical Oncology and Radiotherapy 2012; 17:168-75.

23. Marks LB, Yorke AD, Jackson A, et al. Use of normal tissue complication probability models in the clinic. Int J Radiat Oncol Biol Phys 2010; 76:S10-19.

24. RTOG Radiation Dose Constraints. Available from http://en.wikibooks.org/wiki/Radiation Oncolog y/Toxicity/RTOG.

25. Shaw E, Kline R, Gillin M, et al. Radiation therapy oncology group: Radiosurgery quality assurance guidelines. Int J Radiat Oncol Biol Phys 1993; 27:1231-9.

26. Yoon M, Park SY, Shin D, et al. A new homogeneity index based on statistical analysis of the dose-volume histogram. J Appl Clin Med Phys 2007; 8:2.

27. Monk JE, Perks JR, Doughty D, Plowman PN. Comparison of a micro-multileaf collimator with a $5 \mathrm{~mm}$-leaf-width collimator for intracranial stereotactic radiotherapy. Int J Radiat Oncol Biol Phys 2003; 57:1443-9.

28. Ekambaram V, Velayudham R. Analysis of low-dose level volumes in intensity modulated radiotherapy and 3-D conformal radiotherapy. Int J Cancer Ther Oncol 2014; 2:02032.

29. Esch AV, Tillikainen L, Pyykkonen J, et al. Testing of analytical anisotropic algorithm for photon dose calculation. Med Phys 2006; 33:4130.

30. Bush K, Gange IM, Zavgorodni S, et al. Dosimetric validation of Acuros XB with Monte Carlo methods for photon dose calculations. Med Phys 2011; 38:2208.

31. Rana S. Clinical dosimetric impact of Acuros XB and analytical anisotropic algorithm (AAA) on real lung cancer treatment plans: review. Int $J$ Cancer Ther Oncol 2014; 2:02019.

32. Ojala J. The accuracy of the Acuros XB algorithm in external beam radiotherapy - a comprehensive review. Int J Cancer Ther Oncol 2014; 2:020417. 